\title{
MADEMOISELLE CLAIRON ET SOPHIE ARNOULD VUES PAR LES GONCOURT OU LE THÉÂTRE INTIME DES ACTRICES DU XVIII ${ }^{\mathrm{E}}$ SIÈCLE.
}

\author{
Sophie Marchand
}

En 1855, Edmond et Jules de Goncourt se lancent dans un projet de grande ampleur et envisagent, après avoir scruté la société française pendant la Révolution et le Directoire, d'étudier les vies d'actrices du XVIII ${ }^{\mathrm{e}}$ siècle. S'inscrivant, par sa méthode et ses présupposés théoriques, dans la continuité des précédentes entreprises historiques des deux frères, l'étude du microcosme dramatique s'organise autour des figures emblématiques des actrices les plus célèbres de leur temps, que ce soit par leur talent, leur destinée ou leur personnalité singulière. Les deux frères signent, en 1857, Sophie Arnould d'après sa correspondance et ses mémoires inédits, mais c'est Edmond, resté seul, qui publiera, en 1880, Madame Saint-Huberty d'après sa correspondance et ses papiers de famille, puis, en 1889, Mademoiselle Clairon d'après ses correspondances et les rapports de police du temps. Dans le choix des comédiennes retenues, dans la méthodologie biographique adoptée, dans l'implication des auteurs, transparait l'image particulière que les deux frères se font non seulement des personnalités qu'ils ont choisi d'étudier, mais aussi du théâtre et du XVIII ${ }^{\mathrm{e}}$ siècle. Rien, en effet, ne va de soi dans ces biographies qui, par bien des aspects, surprennent le lecteur familier du théâtre des Lumières. En confrontant les textes des Goncourt à leurs sources et aux autres ouvrages consacrés aux actrices du XVIII ${ }^{\mathrm{e}}$ siècle, en comparant les traitements réservés à la reine de tragédie et à la fille d'opéra, on espère mieux cerner le rapport que ces auteurs entretiennent avec le théâtre et éclairer la place qu'ils occupent dans l'historiographie de cet art. On s'interrogera notamment sur les raisons de cette attention portée aux comédiennes et sur les affinités particulières qui semblent s'établir, dans les textes des Goncourt, entre ces figures de la féminité et le monde dans lequel elles évoluent.

Ce qui frappe, à la lecture des deux biographies majeures de la série, ce sont les différences sensibles dans l'attitude du ou des biographes à l'égard de leurs sujets. Si Sophie Arnould semble jouir de la sympathie des deux frères, le projet qui préside à l'écriture de Mademoiselle Clairon est explicitement iconoclaste. Répondant à un critique qui remettait en question la légitimité de son ouvrage, puisque l'actrice avait laissé des Mémoires, Edmond de Goncourt proteste: «Pardon, tout mon travail a pour but de prouver que ces Mémoires 
ne disent pas la vérité ${ }^{1} »$. Historien et non hagiographe, Goncourt entend fournir un travail objectif, recourant à des sources occultées par une comédienne soucieuse de sa gloire. Sa biographie sera "écrite au moyen du secret des correspondances intimes de Mlle Clairon, à l'aide des divulgations des rapports de police du temps sur sa vie privée »; il s'agit d'écrire "une biographie qui restitue le personnage de la femme dans sa réalité crue, en le terre-à-terre inconnu de son existence d'illustre tragédienne [...], qui la peint, cette originale figure du XVIII ${ }^{\mathrm{e}}$ siècle, avec les jalousies, les intolérances, les tyrannies de son caractère et les faiblesses et les vices et les côtés terriblement humains de la femme, aux lieux et place de l'être conventionnel, de la créature idéalement accomplie et toujours en vedette que nous rencontrons dans le roman de ses Mémoires ${ }^{2}$ ». Il n'y a qu'un pas du portrait objectif au portrait à charge : en dévoilant l'intimité et la vie secrète, Goncourt se lance dans de véritables contre-Mémoires, destinés à démentir «ces Mémoires si peu sincères, si pleins de forfanteries, [...] qui ne laissent rien soupçonner de la Frétillon, telle qu'elle apparait dans les rapports de police et dans les lettres de sa première et libertine jeunesse », "ces Mémoires qui auraient pu être si curieux pour l'histoire du théâtre, des lettres, de la grande société du XVIII ${ }^{\mathrm{e}}$ siècle » mais ne le sont pas $^{3}$. Foncièrement méfiant à l'égard de cette source tendancieuse, Goncourt préfère chercher la vérité dans les documents inédits, et en particulier, dans les lettres autographes, où l'âme de la comédienne s'exprime plus sincèrement ${ }^{4}$. Cette démarche iconoclaste s'inscrit dans le droit fil de celle de Mlle Dumesnil, qui, dès la parution des Mémoires de sa rivale, s'était empressée d'y répondre dans des Mémoires de Mademoiselle Dumesnil, en réponse aux Mémoires d'Hippolyte Clairon. Tout, dans ce texte qui ne laisse guère de place à la biographie de l'actrice, était destiné à dénoncer l'imposture de Clairon, accusée d'avoir non seulement menti sur sa vie et sa personnalité, mais aussi d'avoir détourné le genre des Mémoires au profit d'une entreprise d'autocélébration ${ }^{5}$. La position de Dumesnil n'est cependant pas dominante, et maints biographes de Clairon apprécient ses Mémoires, tout en reconnaissant que le titre ne convient guère à un ouvrage qui n'offre guère d'« événements personnels à l'auteur », mais où l'on trouve « en revanche ce qu'on n'attendait

\footnotetext{
1 Journal, Paris, Robert Laffont, 1989, t. III, p. 410.

2 Edmond de Goncourt, Mademoiselle Clairon, OC, XXXII-XXXIII, Slatkine reprints, Genève, Paris, 1986 , p. 5.

3 Ibid., p. 269-270.

4 « Ce récit des événements depuis la sortie de Mlle Clairon de Fort-L'Évêque jusqu'à la signification de sa retraite, nous le retrouvons plus sincère, plus détaillé, plus intime, dans trois lettres de la tragédienne adressées à Garrick » (Mademoiselle Clairon, p. 149).

5 «Les Mémoires d'une actrice célèbre supposent des aventures galantes, tragiques ou comiques : il n'y a rien de tout cela dans ces prétendus Mémoires. [...] Ce n'est pas que Mlle Hippolyte Clairon manquât de matériaux pour composer de véritables Mémoires, des Mémoires qui eussent été très piquants, très récréatifs [...]. Il faut croire qu'elle a de bonnes raisons pour dérober à nos amusements la partie la plus intéressante de sa vie privée » (Mémoires de Mademoiselle Dumesnil, en réponse aux Mémoires d'Hippolyte Clairon, Paris, chez L. Tenré, 1823, p. 39-40).
} 
pas, des réflexions nobles et touchantes, une morale pure, raisonnable, religieuse, des conseils sur l'éducation des femmes $»^{6}$.

Mais, dans sa démarche historique, Edmond retient de préférence, parmi les témoignages sur la vie de l'actrice, les sources à charge. Le crédit qu'il accorde au point de vue de Dumesnil est, à cet égard, sans ambiguïté. Lorsque s'élève entre les deux comédiennes une dispute sur la réalité d'une anecdote, il prend parti pour Dumesnil contre Clairon 7 . C'est à Dumesnil également qu'il emprunte le portrait peu flatteur d'une Clairon orgueilleuse et mauvaise camarade $^{8}$, n’hésitant pas à affirmer que « depuis que le théâtre existe, aucune femme de théâtre [...] n'a poussé aussi loin l'irrespect du talent et de la personne des auteurs dramatiques ${ }^{9} »$. Certes, cette image de l'actrice existait déjà plus ou moins chez les biographes hagiographes, forcés de reconnaitre que la mauvaise réputation de Clairon n'était pas uniquement imputable à la jalousie suscitée par son talent ${ }^{10}$, mais Goncourt la radicalise en se faisant presque toujours le porte-voix des adversaires de la comédienne ${ }^{11}$.

Goncourt va encore plus loin que Dumesnil dans son entreprise démystificatrice, en recourant à une source d'information unanimement décriée par les biographes de Clairon, un roman satirique et libertin prétendant relater les frasques amoureuses de la jeune actrice, roman anonyme intitulé Histoire de Mademoiselle Cronel dite Fretillon, actrice de la Comédie de Rouen, écrite par elle-même ${ }^{12}$ et publié en 1740 par un prétendant éconduit. Cette Histoire de Frétillon apparaît, dès sa parution, comme un "grossier pamphlet», "mauvais petit roman obscène sans en être moins ennuyeux ${ }^{13} »$. Dumesnil elle-même reconnait (par solidarité d'actrice ?) qu'« on ne saurait être d'un autre avis que Mlle Hippolyte sur L'Histoire de Frétillon », qualifiant l'ouvrage de « libelle dégoûtant», non sans ajouter perfidement: "Nous ne croyons donc pas à cette rhapsodie; mais nous ne croyons pas non plus que Mlle Hippolyte eût osé publier ses Mémoires il y a vingt ans ${ }^{14} »$. Goncourt, moins pudibond, ne répugnera pas à puiser à cette source: «Tout en n'employant pas comme autorité historique l'Histoire de Mlle Frétillon, pour les aventures de la jeune Clairon, je crois que l'auteur du

\footnotetext{
${ }^{6}$ Voir Pierre-Édouard Lemontey, "Notice sur Mlle Clairon », extrait de la Revue encyclopédique (60 cah., t. XX), décembre 1823. Notice lue devant l'Académie française le 4 novembre 1825, destinée à faire partie de la Galerie française, p. 14).

7 C'est ce qui se produit à propos d'un entretien qu'aurait eu, au début de sa carrière, Clairon avec le duc de Gesvres (Mademoiselle Clairon, p. 38 et Mémoires de Mademoiselle Dumesnil, p. 301).

8 Mademoiselle Clairon, p. 158.

9 Ibid., p. 163. Là encore, il reprend le point de vue de Dumesnil (Mémoires de Mademoiselle Dumesnil, p. 115).

${ }^{10}$ Voir la «Notice sur la vie de Mademoiselle Clairon» qui accompagne les Mémoires de l'actrice (p. xliii).

${ }^{11}$ Évoquant la querelle qui oppose Clairon à Lekain, il parle de «cabale effroyable » contre un " grand acteur », et dénonce la vanité des motifs de Clairon (Mademoiselle Clairon, p. 68)

${ }^{12}$ La Haye, Aux dépens de la Compagnie, MDCCXL.

13 "Notice sur la vie de Mademoiselle Clairon», Mémoires, p. v. La même condamnation s'exprime chez Lemontey, (« Notice sur Mlle Clairon», art. cit., p. 3).

${ }^{14}$ Mémoires de Mademoiselle Dumesnil, p. 218.
} 
pamphlet donne des détails assez véridiques sur le théâtre en province, et je me sers de ces détails ${ }^{15}$ ». Placé sur le même plan que les rapports de police, ce roman permet à Goncourt d'atteindre la vérité de l'actrice, cachée par les biographies officielles. Mademoiselle Clairon s'annonce donc comme un portrait à charge, visant en réalité moins l'actrice que la mythologie du génie qui accompagne, au XVIII ${ }^{\mathrm{e}}$ siècle, l'essor éditorial des textes consacrés aux acteurs et leur réception. Cette volonté démystificatrice, qui vise certes, en premier lieu, une certaine conception du métier d'acteur, s'appuie sur une réflexion concernant l'écriture de l'histoire.

Il s'agit, pour Goncourt, d'anéantir la mythologie mise en place par les Mémoires de Clairon. Pour ce faire, il faut revenir à la vérité, en se délestant de la légende véhiculée depuis un siècle par des anecdotes au symbolisme chargé. Goncourt n’hésite pas à écorner le récit des origines fourni par la comédienne, qui prétend, étant née durant le carnaval, avoir été baptisée par un curé habillé en Arlequin et son vicaire habillé en Gille ${ }^{16}$. Si les biographes officiels reprennent sans broncher cette anecdote, s'enthousiasmant d'un tel signe du destin ${ }^{17}$, d'autres, plus circonspects, notent qu'«il n'était pas besoin de cette fable pour signaler la vocation qui la fit comédienne ${ }^{18} »$. Dumesnil juge le fait "digne du Roman comique ${ }^{19}$ ", sans le prendre au sérieux, à la différence de Goncourt qui, non content d'invalider l'anecdote, se sent tenu d'y apporter un démenti historique : «Le malheur de l'anecdote, qui voue si joliment l'enfant au théâtre, c'est que Clairon naquit un 25 janvier [il cite l'acte de naissance en note] et jamais le carnaval, jamais les jours gras ne tombèrent en ce mois. Peutêtre bien la grande tragédienne fut-elle baptisée [...] au dessert d'un plantureux repas des Flandres, mais par un curé qui n'était pas en Arlequin, mais par un vicaire qui n'était pas en Gille ${ }^{20} »$. En refusant à la fois la symbolique théâtrale et la narration téléologique, le biographe dément l'idée selon laquelle le monde de la scène serait le terreau naturel de l'actrice, préférant réserver sa prédestination à un autre écrin - celui de la vie galante ${ }^{21}$, et stigmatise ce qui

\footnotetext{
15 Mademoiselle Clairon, p. 20 et 21 ; voir aussi p. 17-18 : «le tableau que fait un roman-pamphlet de la vie des deux femmes n'est pas trop en désaccord avec l'aveu, légèrement voilé, que l'actrice fera plus tard des désordres de sa mère ». L'Histoire de Frétillon n'est peut-être pas entièrement vraie, mais elle est vraisemblable et cela suffit aux yeux d'un Edmond moins soucieux de peindre en Clairon un portrait original que l'incarnation d'un type social et d'une époque.

${ }^{16}$ Clairon, Mémoires, p. 8.

17 "Notice sur la vie de Mlle Clairon », Mémoires, p. v. Voir aussi Amoldiana ou Sophie Arnould et ses contemporaines, par l'auteur du Biévriana [Deville], Paris, Gérard, 1813, p. 101-102.

${ }^{18}$ Lemontey, « Notice sur Mlle Clairon », art. cit., p. 1-2.

19 Mémoires de Mademoiselle Dumesnil, p. 317.

20 Mademoiselle Clairon, p. 10.

${ }^{21}$ En dissociant ainsi l'imaginaire du théâtre de la narration biographique, Goncourt prépare le portrait d'une Clairon plus courtisane qu'actrice de génie. En réalité, ce n'est pas à l'anecdote symbolique qu'il répugne, mais au mythe de l'actrice entièrement déterminée par son art. Il est, à cet égard, significatif qu'évoquant les orgies plus tardives de Clairon, il convoque la mythologie théâtrale en contrechamp du libertinage : «cette maison de la petite rue des Marais,
} 
semble devenu un topos des Mémoires d'acteurs: une naissance marquée du sceau de la singularitée ${ }^{22}$.

$\mathrm{Si}$ le refus de l'anecdote trop belle pour être vraie semble une caractéristique de la méthode historique des Goncourt ${ }^{23}$, il témoigne surtout du désir de peindre une Clairon différente de la légende érigée autour d'elle, par l'actrice elle-même mais aussi par un certain dix-huitième siècle (Voltaire, Diderot, Marmontel entre autres) soucieux de faire de la comédienne l'emblème d'un théâtre réformé selon les principes des Lumières. Cette figuration de l'artiste en Philosophe, Goncourt va s'y attaquer en se lançant dans une démystification hargneuse de la stratégie apologétique mise en œuvre par les Mémoires. Clairon, en effet, n'a de cesse de démentir la réputation de galanterie qui lui fut associée dès ses débuts à la comédie de Rouen. Tout l'ouvrage semble déterminé par l'ambition de dissocier l'actrice de génie de Frétillon. Aussi, non contente de justifier ponctuellement ses liaisons intéressées ${ }^{24}$, se lance-t-elle dans une confession qui frappe par son allure de plaidoyer :

sans entrer dans le détail des erreurs, des malheurs et des plaisirs où mon éducation, la sensibilité de mon âme mon libre-arbitre et l'exemple ont pu m'entraîner, $[\ldots]$ je ne prétends dissimuler aucune de mes fautes, et je conviens que j'en ai fait beaucoup. [...] Mon talent, mon personnel, la facilité de m'approcher m'ont fait voir tant d'hommes à mes pieds, qu'il était impossible qu'une âme naturellement tendre, obligée de se pénétrer sans cesse de ce que les passions ont de plus séducteur, pût se trouver inaccessible à l'amour. Qu'on cesse quelques instants de surveiller les filles les mieux nées, qu'on entr'ouvre seulement la plus petite grille d'un cloître, je serai pleinement justifiée. L'amour est un besoin de la nature : je l'ai satisfait, mais de manière à n'en point rougir. 25

ce logis orgiaque, savez-vous quelle est cette maison? O ironie !». Il s'agit d'une maison autrefois occupée par Racine puis par Lecouvreur » (Mademoiselle Clairon, p. 66-67). La légende théâtrale joue cette fois contre Clairon, et Goncourt, évoquant ces figures illustres rejette l'actrice hors de leur univers. Le monde de Clairon est celui de l'orgie, et la cohabitation symbolique avec ces grandes figures du théâtre ne fait que souligner la chute de l'actrice (chute qui n'est associée à aucun discours moral).

22 Il démentira, de même, la légende forgée autour de la naissance de Sophie Arnould, qui serait née «dans la même alcôve où avait été assassiné l'amiral Coligny» (Sophie Arnould, p. 15). Cependant, l'enjeu du mensonge mythographique semble ici différent : Sophie Arnould pèche par vanité (en femme coquette, elle ment aussi sur son âge - ibid., p. 16), en singeant les naissances illustres de l'aristocratie et en tentant d'inscrire son existence dans l'histoire, mais en aucun cas elle ne tente de s'octroyer une légitimité théâtrale.

${ }^{23}$ Le Journal du 14 juillet 1856 cite un reproche adressé aux deux frères, accusés de recourir à des anecdotes trop peu connues, au mépris des anecdotes d'ana (voir Paule Adamy, "Mais pourquoi les opéras finissent-ils? ou la série des Actrices du XVIII siècle », Cabiers Goncourt, 5, 1997, p. 186).

${ }^{24}$ « Ce moment, qui ne présente au premier aspect que l'idée du libertinage, est peut-être le plus noble, le plus intéressant, le plus frappant de ma vie » (Clairon, Mémoires, p. 26).

${ }^{25}$ Ibid.,p. 46-47. 
À cette justification qui désigne la galanterie par le lexique de la sensibilité la plus philosophique et évacue le libertinage au profit d'une dénonciation des disconvenances sociales qui ne déparerait pas dans la bouche de la Marceline de Beaumarchais, s'ajoutent la présence dans les Mémoires d'une partie intitulée «Réflexions morales » (qui stigmatise notamment la vénalité féminine ${ }^{26}$ ) ainsi que la proclamation d'une ambition édifiante ${ }^{27}$.

À l'inverse d'autres biographes, enclins à célébrer en cette pécheresse repentante l'avènement d'une moraliste d'autant plus crédible ${ }^{28}$, Edmond refuse de se laisser séduire par la pose apologétique. Son ton, violemment démystificateur, appelle à «donner la parole à Frétillon, et place à l'invraisemblable historiette que l'invraisemblable vierge du théâtre de Rouen a le sang-froid d'offrir au public ${ }^{29} »$. Là où Clairon jouait des topoi de l'esthétique sentimentale (vertu persécutée par l'arbitraire parental, mariage forcé, préséance du sentiment sur l'intérêt...), Goncourt, citant les Archives de la Bastille, met à nu le libertinage d'un siècle et d'un milieu essentiellement libertins ${ }^{30}$. Émerge alors l'image d'une "auguste concubine ${ }^{31}$ », et Goncourt n'affecte d'être magnanime que pour mieux couronner son entreprise iconoclaste: "Arrêtons ici le dénombrement des amants payants de celle qui dit dans ses Mémoires n'avoir jamais à rougir de ses amours, et qui défie qu'on lui cite, dans toute sa vie, un marché honteux, "un seul homme qui l'ait payée..." ${ }^{32}$.

En réalité, cette démarche vise moins Clairon que la condition d'actrice en général et le propos de Goncourt prend vite une teinte sociologique : «Les rapports de police ont apporté une triste lumière sur les faiblesses des femmes de théâtre, non des ballerines et des déclamatrices des petits théâtres, mais des illustres, des célèbres des grands théâtres, mais des plus renommées actrices de la Comédie-Française. La prostitution de ces femmes-là $[\ldots]$ ne diffère pas de celle des impures de bas étage ${ }^{33} »$. La chute de l'icône s'inscrit dans un combat

\footnotetext{
26 Ibid., p. $166-167$.

27 Ibid., p. 49.

${ }^{28}$ L'éditeur des Mémoires, tout en reconnaissant la conduite galante de la comédienne (Notice, p. 47), la met sur le compte des mœurs théâtrales (p. vii), invitant ainsi à l'indulgence et se félicitant de voir en Clairon, "malgré la dissipation qui l'entraîna pendant une partie de sa vie », un « caractère $[\ldots]$ sérieux, grave, élevé » (Notice, p. 162).

${ }^{29}$ Mademoiselle Clairon, p. 25.

30 Semblable dénonciation était déjà présente chez Mlle Dumesnil (p. 225), qui demandait: "Ces Mémoires ne seraient-ils pas un essai que vous auriez voulu faire sur la crédulité humaine ? ", appelant sa consœur à plus de franchise : "Vous n'aviez qu'un parti à prendre, celui de jouer franchement et loyalement [...] le rôle d'une femme galante revenue de ses erreurs [...]. Vous avez mieux aimé jouer le personnage le plus ridicule pour la fameuse Clairon, celui de prude » (Mémoires de Mademoiselle Dumesnil, p. 228-229).

31 Mémoires de Mademoiselle Dumesnil, p. 355. On retrouvera au XIX e siècle, cette image chez Arsène Houssaye (La Comédie Française, 1680-1890, Paris, Baschet, 1880, p. 140-141).

32 Mademoiselle Clairon, p. 63.

33 Mademoiselle Clairon, p. - 64.
} 
plus vaste, et Clairon, qu'on veut emblématique de ses consœurs, perd sa singularité d'actrice de génie. Sans acquiescer naïvement à l'angélisme moral des Mémoires, force est pourtant de reconnaitre que la figuration de Clairon en courtisane de haut vol n'est guère plus satisfaisante et véridique que celle de Clairon moraliste. Il demeure indéniable que l'actrice prit à l'histoire du théâtre et de la reconnaissance de celui-ci (qu'elle soit artistique, morale ou sociale) une part importante, dont témoigne notamment la lutte qu'elle mena, dans les années 1760, pour l'abrogation de l'excommunication des comédiens ${ }^{34}$. Ces combats, que Goncourt ravale au rang de manifestations de l'orgueil de la « reine de théâtre ${ }^{35}$ ", révèlent l'engagement de Clairon dans un monde théâtral qui ne saurait se réduire au boudoir des actrices.

Mais ce théâtre-là n'est, de toute évidence, pas celui qui intéresse les Goncourt biographes. Si le portrait de Clairon permettait encore d'en douter, sa confrontation avec celui de Sophie Arnould le fait apparaittre de manière éclatante. En contrepoint de l'entreprise iconoclaste de Mademoiselle Clairon, Sopbie Arnould déploie l'intimité d'une fille d'opéra peinte au naturel, révélant ce que représentent les actrices aux yeux des deux frères. Il n'est pas anodin que ce portrait ouvre la série des Actrices du XVIII siècle et soit le seul réalisé en commun. Commencer par Sophie Arnould n'allait pas de soi: certes la cantatrice avait marqué son temps, certes les ouvrages consacrés à sa vie ne manquaient pas ; mais plus que de la carrière de l'actrice, ceux-ci perpétuaient la mémoire de ses bons mots, célébrant une gloire gagnée dans les coulisses et les salons plutôt que sur la scène. Clairon, en revanche, incarnait pour le XVIII ${ }^{\mathrm{e}}$ siècle l'actrice de génie, associée aux mutations d'un art en pleine redéfinition et en voie d'autonomisation. Faisant de Sophie Arnould l'actrice par excellence du XVIII ${ }^{\mathrm{e}}$ siècle, l'ordre de rédaction des biographies d'actrice trahit les présupposés qui animent l'entreprise historique des deux frères et leur conception du théâtre.

La cantatrice offre à leur projet un support bien plus favorable. Les Goncourt s'extasient devant les lambeaux retrouvés d'hypothétiques Mémoires de Sophie Arnould, qui, à la différence des Mémoires apprêtés de Clairon, auraient la «même authenticité historique » que les documents vrais : «c'est le tour et l'esprit de Sophie Arnould et son ton et son accent. Cette voix [...], n'est-ce pas le caractère et le goût propre des mémoires d'une comédienne qui se confesse? Sophie n'affiche-t-elle pas, dans une lettre à Lauraguais [...],

\footnotetext{
34 C'est à l'initiative de la comédienne que l'avocat Huerne de La Mothe publie un ouvrage intitulé Libertés de la France contre le pouvoir arbitraire de l'excommunication. Ouvrage dont on est spécialement redevable aux sentiments généreux et supérieurs de Mademoiselle CLAI** (Amsterdam, MDCCLXI). Citant une lettre de la comédienne où elle se présente comme «née citoyenne, élevée dans la religion chrétienne catholique » et « soumise aux décisions de l'Église » (p. xv), il en fait le héraut de son combat. Ses adversaires ne s'y tromperont pas, préférant répondre à l'actrice qu'à l'avocat (voir les Lettres historiques et critiques sur les spectacles, adressées à Mlle Clairon, Avignon, chez les Libraires associés, MDCCLXII., et particulièrement la lettre 10, p. 176-177).

35 Mademoiselle Clairon, p. 120.
} 
l'intention d'écrire l'histoire de ses amours $?^{36} »$. Parce qu'elle se peint telle qu'elle est, actrice en déshabillé délestée de sa parure de scène, Sophie Arnould apparaît comme le parfait sujet de la biographie intime à laquelle aspirent les Goncourt. Car les vies d'actrices s'inscrivent dans la même veine que les Histoires de la Société française, Les portraits intimes du XVIII siècle, l'Histoire de MarieAntoinette ou les autres biographies féminines de cette époque, textes où les deux frères, au mépris de la tradition historique, recherchent «l'anecdote, le coin intime des hommes et des choses ${ }^{37} »$. Pour parvenir à cette intimité, ils privilégient de nouvelles sources, en particulier «les lettres familières », dans lesquelles l'«abandon de l'amitié, [la] causerie de l'intimité n'admettent ni faussetés ni détour » et où « les pensées les plus secrètes [se] trahissent, l'esprit et le cœur [se] montrent sans déguisement ${ }^{38} »$. Dans ces documents se révèle la vérité des actrices, qui se dérobe dans les Mémoires. Goncourt se réjouit donc de la découverte d'une correspondance de Clairon avec Larive qui montre "l'actrice, dans le train train de la vie tranquillement intime, dans le repos apaisé et les occupations de son coin du feu [...]; sur ce fond d'intérieur, hélas! fermé, perdu, irretrouvable des comédiennes du passé39 ». Ces «documents intimes qu'une époque laisse derrière elle pour être sa confession et sa résurrection ${ }^{40} »$ redessinent les contours de la science historique, faisant apparaittre un autre type de personnage. Soucieux non du héros ou du grand homme, mais de "l'homme qui était cet homme d'état, cet homme de guerre, ce poète, ce peintre, ce grand homme de science ou de métier", "l'âme qui était en cet acteur, le cœur qui a vécu derrière cet esprit », l'historien moderne " [pratique], dans une amoureuse familiarité, et dans leurs grâces les plus secrètes, les âmes charmantes d'un grand siècle ». C'est ce que les deux frères nomment «histoire intime $»^{41}$. Cette démarche, qui dévoile la femme sous le personnage, est à mille lieues de celle de Clairon dans ses Mémoires, ou de celle de son éditeur, qui prévient : « nous nous occuperons plus [...] des événements de sa carrière théâtrale que de ceux de sa vie privée ; les premiers appartiennent plus particulièrement à l'ouvrage que nous faisons paraittre ${ }^{42} »$. Loin de toute démarche hagiographique et de toute ambition commémorative, la biographie " résurrectrice» des Goncourt privilégie «le petit bout de la lorgnette », les hissant au rang d'«archéologues du détail » ${ }^{43}$. Soucieuse de raconter l'histoire de ses amours, marquant son siècle d'une empreinte de futilité et de la grâce volatile de son esprit, Sophie Arnould s'inscrit parfaitement dans cette histoire.

Mais ce n'est pas seulement la femme intime s'offrant aux regards qui séduit les Goncourt en Sophie Arnould : c'est aussi l'actrice au naturel, la fille

\footnotetext{
36 Sophie Arnould, p. 6-7.

${ }^{37}$ Histoire de la société pendant le Directoire, OC, t. XL, p. 156.

38 Sophie Arnould, p. 11.

${ }^{39}$ Mademoiselle Clairon, p. 183-184

${ }^{40}$ Histoire de la société française pendant la Révolution, OC, t. XXIII, p. v-vi.

${ }^{41}$ Portraits intimes du XVIII siècle, OC, t. XXXVIII-XXXIX, p. 7-12.

42 Clairon, Mémoires, p. iv.

${ }^{43}$ M. Delon, «Les anecdotiers du XVIII ${ }^{\mathrm{e}}$ siècle », Magażine littéraire, 269, 1989, p. 48.
} 
d'opéra assumant son statut sans hypocrisie et le proclamant au grand jour, la démystificatrice caustique du mythe théâtral. Dans la dénonciation de la stratégie apologétique mise au point par Clairon, Sophie Arnould fait figure de précurseur et d'adjuvant des Goncourt. Dès le XVIII ${ }^{\mathrm{e}}$ siècle, une anecdote célèbre met aux prises les deux actrices : «Mlle Clairon [...] disait avec emphase que le roi était maittre de sa vie et de sa fortune, mais non de son honneur. Vous avez raison, observa Sophie, où il n'y a rien, le roi perd ses droits ${ }^{44}$ ». Dans les faux Mémoires qu'il prête à Sophie Arnould, Lamothe-Langon joue, lui aussi, de l'opposition entre les deux comédiennes. Évoquant Clairon, sa Sophie fictive raconte: «Je voulais me lier avec cette superbe reine, cela ne fut pas possible; en menant la vie de Messaline, elle voulait qu'on lui portât respect comme si elle eût été la mère de Gracques ; ce ton ampoulé, ridicule, ne me convenait $\operatorname{pas}^{45}$ ». Âme franche assumant la corruption du monde des théâtre, Sophie Arnould marque son temps par ses saillies cyniques, dont les recueils d'anecdotes se font le complaisant vecteur ${ }^{46}$. Les Goncourt sont particulièrement sensibles à cette démystification et citent un extrait de l'Arnoldiana: «Mlle Arnould était reçue partout parce qu'elle se mettait au niveau de son état et ne voulait être qu'une actrice et, comme on disait alors, une fille d'opéra. Je me souviens d'avoir été chez elle à un brillant souper [...]. Ces demoiselles faisaient les dames; on eût dit que c'étaient des princesses. Mlle Arnould, d'une voix ferme, en parlant d'une chose qui convenait à des femmes de qualité ajouta : "Mais pour nous, Mesdames, nous sommes des p... cela est différent" ${ }^{\prime 47} »$. Cette reconnaissance va parfois jusqu'à prendre l'allure d'une profession de foi ou d'une revendication identitaire. À un «censeur atrabilaire » qui «critiquait [...] le luxe scandaleux des courtisanes et des actrices », l'actrice répond ainsi : «Eh, monsieur, laissez- les jouir de la perte de leur réputation ${ }^{48} »$. La démarche est exactement inverse à celle de Clairon et la confrontation des deux actrices et du traitement qui leur est réservé par leurs biographes permet de mettre au jour l'idée que les Goncourt, comme une bonne partie du XIX ${ }^{\mathrm{e}}$ siècle, se font des actrices et du monde théâtral.

La vérité des actrices, en effet, n'est pas à chercher du côté de l'art, mais dans une nature féminine qui les prédisposerait au rôle de femme galante, confondu avec celui de comédienne. Avant d'être une actrice (ou peut-être parce qu'elle est une actrice), la comédienne est une courtisane. Le portrait de

44 Esprit de Sophie Amould, Paris, chez F. Louis, libraire, 1813, p. 38. Voir aussi Arnoldiana, p. 138. L'anecdote est évidemment démentie par les Mémoires de Clairon (Notice, p. lviij).

45 Mémoires de Mademoiselle Sophie Arnould, recueillis et publiés par le Baron de Lamothe-Langon, Paris, Allardin, 1837, t. II, p. 13. Dumesnil, elle aussi, opposait les attitudes de Clairon et Sophie Arnould (Mémoires, p. 250).

46 « Le duc de Praslin lui demandait des nouvelles d'une actrice de l'opéra dont il cherchait à se rappeler le nom. C'est une jeune personne, lui dit-il, dont le nom finit en AIN. - Ah! Monsieur le duc, vous ne le trouverez pas, tous nos noms finissent comme cela » (Esprit de Sophie Arnould, p. 24 ; Arnoldiana, p. 147). On pourrait en citer bien d'autres.

47 Sophie Arnould, p. 56.

48 Arnoldiana, p. 158. Une autre anecdote va dans le même sens (Esprit de Sophie Arnould, p. 34). 
Sophie Arnould s'ouvre sur l'évocation de ces «rares créatures » et l'annonce de « la dernière venue, la dernière peut-être, la sœur cadette de Ninon, la seule courtisane de l'âge d'or des filles : Sophie Arnould ${ }^{49} »$. Clairon fut, elle aussi, comparée à Ninon de Lenclos ${ }^{50}$ et nombreux sont les textes du XIX ${ }^{\mathrm{e}}$ siècle qui voient dans les comédiennes du XVIII ${ }^{\mathrm{e}}$ siècle de nouvelles Aspasie, Laïs ou Phryné, substituant à la mythologie de l'art celle de l'amour et de la beautéc ${ }^{51}$. Dans le compte rendu qu'il rédige de Sophie Arnould des Goncourt, Barbey d'Aurévilly n'envisage pas l'actrice mais la courtisane dont le nom est un «talisman pour toutes les imaginations de ce temps qui a toutes les curiosités de la corruption et toutes les corruptions de la curiosité ${ }^{52} »$. Cette assimilation de l'actrice à la femme galante est indissociable de l'imaginaire fantasmatique cristallisé par l'institution de l'Opéra, qui apparaît, au XVIII siècle comme au $\mathrm{XIX}^{\mathrm{e}}$, comme un lieu voué au libertinage plus encore qu'à l'art ${ }^{53}$. Cette image des actrices était présente dans l'Histoire de Frétillon, où, après avoir recueilli l'avis d'un intendant qui lui conseillait de se faire courtisane, la jeune Cronel, effrayée par la mauvaise réputation attachée à cet état, préférait, sur les conseils de sa mère, se tourner vers la scène, persuadée que «le théâtre est un point d'optique avantageux pour une fille que la fortune a négligée » et que «le goût bizarre des hommes pour les chimériques princesses du Théâtre est un fonds inépuisable de trésors qui sert au luxe des Armide et des Andromaque ${ }^{54} \gg$. On connait la fortune romanesque de cette topique, dans un XIX ${ }^{\mathrm{e}}$ siècle qui associe naturellement scène du théâtre et scène de la galanterie.

Les vies d'actrices sont donc avant tout des vies de femmes, et de femmes galantes, et il n'est, dans cette perspective, guère étonnant de voir, dans les biographies des Goncourt, la figure de l'artiste bien souvent éclipsée au profit de la peinture du caractère féminin. Commentant les reproches que lui adresse un critique à propos de Mademoiselle Clairon, Edmond opère une mise au point: "la femme ne l'intéresse pas, il n'y a que l'actrice; moi, la femme m'intéresse autant que l'actrice ${ }^{55}$ ». S'intéresser à la femme, c'est considérer l'actrice en dehors du théâtre, sur la scène du monde. Plus que le succès de l'actrice, c'est sa dimension de femme publique qui nourrit la biographie: "personnage, dont les faits, les gestes, les fantaisies [occupent] Paris », Clairon se voit acclamée en même temps que le roi $^{56}:$ «la Frétillon d'autrefois est devenue la grande personnalité de la Comédie-Française,

\footnotetext{
49 Sophie Arnould, p. 13.

${ }^{50}$ Lemontey, art. cit., p. 10.

${ }^{51}$ Voir Houssaye, Les Femmes du diable, Paris, Michel Lévy frères, 1867, p. 261-262.

52 Barbey d'Aurevilly, Le Pays, 4 juin 1857, reproduit dans Articles inédits (1852-1884), publiés par Andrée Hirschi et Jacques Petit, Annales littéraires de l'université de Besançon, 138, Les Belles-Lettres, Paris, 1972, p. 26-27.

53 Voir Arnoldiana, notice sur l'opéra, p. 26-27.

54 Histoire de Mademoiselle Cronel dite Fretillon, p. 34.

55 Journal, 5 avril 1890, t. III, p. 410. On observe le même parti pris dans l'Histoire de MarieAntoinette : «ils ne donnent pas pour le portrait de la Reine la figure de convention [...]. Ils montrent une femme, une femme du dix-huitième siècle » (OC, t. XXIII, p. 5).

56 Mademoiselle Clairon, p. 60 et 116.
} 
l'actrice au renom populaire en même temps que la femme à l'influence de laquelle ses camarades doivent auprès des ministres, des secrétaires d'état, des gentilshommes de la chambre, faveurs, grâces, $\operatorname{argent}^{57}$ ». Il en va de même pour Sophie Arnould: Arsène Houssaye note, à propos de l'enlèvement de l'actrice par le comte de Lauraguais: "Sophie était déjà à la mode dans le monde des passions profanes. Sa renommée resplendit d'un vif éclat; on ne l'avait comparée qu'à Orphée, on la compara à Sapho et à Ninon ${ }^{58}$ ».

Transportées de la scène de théâtre sur la scène du monde, les comédiennes du XVIII ${ }^{\mathrm{e}}$ siècle deviennent actrices de leur propre vie. Edmond évoque, dans Mademoiselle Clairon la " petite nature dramatique » de l'enfant, qui lui fait tenir tête à sa mère ${ }^{59}$. Et dans Sophie Arnould, c'est sur la scène sociale, celle des blagues de Lauraguais et de Sophie, dont est victime le prince d'Hénin, que se joue «la meilleure comédie de l'année et la plus applaudie ${ }^{60}$ ». Et dès lors que l'amour n'est qu'un jeu, l'actrice n'est-elle pas naturellement comédienne? Derrière ces métaphores et ces transferts, se fait jour l'idée que l'état de comédienne serait bien moins un métier qu'une émanation de la nature féminine. La vie des actrices échappe ainsi à la singularité : Clairon, comme Sophie Arnould, est condamnée à se fondre dans le moule d'un archétype qui relève d'une détermination autant sociologique que psychologique, voire physiologique. D’où ces généralisations ${ }^{61}$ qui, dans les biographies d'actrices par les Goncourt, estompent la peinture du caractère au profit de la description quasiment naturaliste d'une condition. Le comble de l'assimilation entre complexion féminine et vocation théâtrale apparaîtra dans Sophie Arnould, où, évoquant les amours féminines de la cantatrice, Edmond note : "Le goût des chanteuses pour leur sexe, c'est vraiment très particulier comme on le rencontre, ce goût! dans tous les temps et dans tous les pays ", ajoutant: «Dans le roman de La Faustin, j’ai signalé les rapports, constatés par la médecine, des organes vocaux avec les organes génitaux de la femme, et le développement de ces derniers chez toutes les chanteuses, déclamatrices, etc. ${ }^{62} »$. La vérité des actrices, échappant à l'art, serait purement physiologique.

La généralisation typologique mise en place par les Goncourt délimite les contours d'un archétype qui ne différencie pas l'actrice en tant qu'artiste de la comédienne comme femme, insérée dans un milieu et une condition particulière. Ce type social, dont la réalité se veut attestée par la revendication d'une méthodologie historique, se retrouve dans maints romans du temps sur

\footnotetext{
57 Ibid., p. 116. Comme le constate P. Adamy, «Goncourt a une double ambition : montrer la femme publique - au double sens du mot: une fille publique ou courtisane et une actrice vouée au public - et la femme privée, dans ce qu'il appelle son intime » (art. cit., p. 197).

${ }^{58}$ Les Femmes du diable, p. 274.

59 Mademoiselle Clairon, p. 14.

60 Sophie Arnould, p. 86.

${ }^{61} \mathrm{La}$ mère de Clairon ne fait que «suivre l'exemple de toutes les mères d'actrices du temps » (Mademoiselle Clairon, p. 17-18); "Sophie eut même en ces années, ainsi que les femmes de théâtre en ont de temps en temps, un coup de cœur pour un inférieur » (Sophie Arnould, p. 51).

${ }^{62}$ Sophie Arnould, p. 82
} 
les actrices et dans le goût contemporain pour les ouvrages voués à satisfaire la curiosité du public sur les coulisses et la vie secrète des théâtres. S'ils sont porteurs de vérité sociologique, ces portraits ne peuvent cependant manquer de décevoir (ou du moins de surprendre) l'historien ou le connaisseur du théâtre du XVIII ${ }^{\mathrm{e}}$ siècle, étonné de voir à ce point évacuée la dimension proprement théâtrale de ces vies d'actrice.

Du théâtre, en effet, que reste-t-il dans cette mise à nu de l'intimité des actrices? D'emblée, le lecteur est frappé par la place laissée dans ces biographies à tout ce qui n'appartient pas proprement à l'activité théâtrale. Loin de se focaliser sur la carrière de leurs sujets, les portraits d'actrice n'y consacrent qu'une portion de l'ouvrage (particulièrement dans le cas de Sophie Arnould), alors que les textes consacrés aux acteurs au XVIII ${ }^{\mathrm{e}}$ siècle développaient abondamment la description des rôles et des techniques de jeu. Une attention toute particulière est, par ailleurs, accordée à la vie des actrices hors du théâtre ${ }^{63}$ et après leur retraite ${ }^{64}$. Curieusement, c'est alors qu'elle a quitté la scène que, l'idole détrônée laissant percevoir la femme, Clairon semble enfin pouvoir jouir d'un peu d'admiration et d'empathie de la part de son biographe. Les Goncourt s'attardent alors sur ces « années silencieuses [...] où rien ne parle plus de ces vies, tout à l'heure si retentissantes de publicité6 ${ }^{65} »$. De même, c'est sur «la reine d'Opéra $[. .$.$] devenue une quasi paysanne» que$ s'émeuvent les deux frères. Cette insistance sur la vieillesse des actrices identifie le projet biographique à la description d'une trajectoire exemplaire (splendeurs et misères des courtisanes $\left.{ }^{66}\right)$, relevant d'un topos romanesque qui n'est pas spécifiquement théâtral.

Outre ces partis pris structurels qui minorent, dans la destinée féminine, la part de l'artiste, ce qui frappe encore, c'est le refus des biographes d'adopter vis-à-vis de leurs sujets une attitude admirative ou excessivement laudative. À la différence de Diderot s'extasiant dans Le Paradoxe sur le comédien devant la sublime Clairon, "cette incroyable magicienne ${ }^{67}$ », Goncourt minimise souvent le talent de l'actrice. Il insiste sur ses débuts laborieux à l'opéra $^{68}$, souligne la faiblesse de sa voix ${ }^{69}$, nuançant ainsi les témoignages élogieux que son souci du document historique l'oblige à citer (le Mercure de France, la Lettre à Madame la marquise V.... de G... sur le début de Mademoiselle Clairon à la Comédie Française ${ }^{70}$ ). Écornant la légende, il ne manque pas de

${ }^{63}$ Comme l'a bien remarqué P. Adamy (art. cit., p. 212-213).

64 Après la retraite de Clairon, sa biographie se poursuit sur 39 chapitres, celle de Sophie Arnould sur 38.

${ }^{65}$ Mademoiselle Clairon, p. 244

${ }^{66}$ Voir Arsène Houssaye, La Comédie Française, p. 143. Barbey d'Aurevilly félicite les Goncourt $\mathrm{du}$ «foudroyant contraste » et de la «magnifique antithèse » ainsi créés (critique de Sophie Arnould, Le Constitutionnel, 18 septembre 1877, Les CEuvres et les hommes, t. XIII, Slatkine reprints, Genève, 1968, p. 308).

${ }^{67}$ Diderot, Paradoxe sur le comédien, Paris, GF Flammarion, 2000, p. 96.

68 Mademoiselle Clairon, p. 35.

${ }^{69}$ Ibid., p. 34.

70 Ouvrage publié à La Haye, MDCCXLIV. 
souligner le scandale provoqué par la réception de Clairon à la ComédieFrançaise, et la cabale menée contre elle par ses confrères ${ }^{71}$. Il s'étonne, enfin, de trouver, dans la correspondance de Voltaire tant «de louanges énormes, [...] de compliments hyperboliques » adressés à l'actrice ${ }^{72}$. De toute évidence, la Clairon de Goncourt n'est pas celle qu'a applaudie le XVIII' siècle et qu'ont encensée les dramaturges-philosophes. Goncourt tente même de minorer la portée novatrice de son jeu, imputant sa volonté d'imposer une action naturelle à sa jalousie envers Mlle Dumesnil ${ }^{73}$ et la qualifiant de «tentative $[. .$.$] pas bien$ hardie $^{74} »$. Reprenant une idée énoncée par Dumesnil, il crédite Marmontel de l'intuition de la réforme du jeu revendiquée par Clairon ${ }^{75}$. Mais cette stratégie ne tient pas, et Goncourt finit par s'incliner à son tour devant le génie d'une Clairon parvenue à sa maturité artistique ${ }^{76}$. Reconnaissant en elle l'origine de ce " qui devrait être le catéchisme dramatique des acteurs et des actrices de notre siècle ${ }^{77}$ ", il loue l'autorité avec laquelle elle parle de son art. On remarque cependant qu'il n'accorde que peu de place à l'exposé des théories de l'actrice, qui occupent pourtant une grande partie de ses Mémoires et en sont la raison d'être $^{78}$. La place dévolue à la description du talent artistique de Sophie Arnould est encore plus mince: les Goncourt se contentent de vanter le naturel de la chanteuse, plaçant son art sous le signe de la nature plutôt que de la technique ${ }^{79}$.

Également révélateur de cette volonté de détourner le propos d'un théâtre conçu comme art et technique est le parti pris de ne pas faire figurer dans les biographies un certain nombre d'anecdotes à portée théorique. Diderot avait fait, dans Le Paradoxe sur le comédien, de Clairon le modèle de l'acteur de sang-froid et d'étude, soumis au modèle idéal plutôt qu'à ses passions. Cette Clairon n'apparaît guère dans le texte de Goncourt, qui développe, dans les biographies, à propos des actrices, une conception bien peu diderotienne. On observe le même processus à propos de Sophie Arnould, qui, elle aussi, figurait dans le Paradoxe. Alors qu'ils citent de nombreuses anecdotes tirées des ana consacrés à la chanteuse, les Goncourt en omettent significativement une qui offrirait de l'actrice une image différente de celle de la courtisane spirituelle : «Le baron de Grimm rapporte, dans sa correspondance, que cette Sophie, si touchante au théâtre, employait les moments où elle faisait pleurer et frémir toute la salle à dire tout bas des folies aux acteurs qui se

\footnotetext{
${ }^{71}$ Mademoiselle Clairon, p. 36. La cabale était motivée par la réputation sulfureuse de l'actrice plus que par la mise en cause de son talent.

72 Ibid., p. 122.

73 Ibid., p. 74.

${ }^{74}$ Ibid., p. 73.

75 Ibid., p. 74. Voir Mémoires de Mademoiselle Dumesnil, p. 118.

76 Mademoiselle Clairon, p. 77.

${ }^{77}$ Ibid., p. 85.

78 Clairon, Mémoires, p. 280.

79 «Son âme a fait sa voix, et son visage est le portrait de son âme» (Sophie Arnould, p. 41). Une fois de plus, la femme fait -et éclipse- l'artiste.
} 
trouvaient en scène avec elle ; et, lorsqu'il lui arrivait de tomber évanouie entre les bras d'un amant au désespoir, elle ne manquait guère, pendant que le parterre s'extasiait, de dire à l'acteur éperdu: Ab! mon cher Pillot, que tu es laid $!^{80} »$. Alors qu'au XVIII ${ }^{\mathrm{e}}$ siècle l'intérêt pour les comédiens semblait motivé par une réflexion théorique sur l'art dramatique, les biographies d'actrices par les Goncourt opèrent une sortie du théâtre.

Pas plus qu'ils ne semblent voués à susciter ou manifester une pensée de l'art, ces textes ne sont destinés à fournir sur la carrière des actrices une information positive. Certes les Goncourt compulsent les registres des théâtres, dressent des listes de rôles et de pièces, mais la sécheresse de ces données objectives est bien décevante, les biographes se révélant, sur ce point, particulièrement laconiques ${ }^{81}$. On est bien loin de l'intérêt manifesté à l'égard des objets et des images des actrices, de cette curiosité de collectionneur qui informe la démarche historique d'Edmond. Cet intérêt semble cependant renaitre dès lors que la biographie aborde l'activité professionnelle des actrices non dans sa dimension "poétique » (rôles, conceptions du jeu...), mais sous l'angle de la vie théâtrale, dans ce qu'elle a de plus concret. On voit alors les deux frères tenir les comptes des appointements et gratifications, comme si ces informations appartenaient, à leurs yeux, davantage à la réalité du théâtre ${ }^{82}$. Dans le domaine théâtral, comme dans celui de l'histoire, la démarche des Goncourt privilégie le petit, le concret, les sources inattendues, à milles lieues de la légende mais au plus près de l'intimité des sociétés. Si le théâtre intéresse les Goncourt, ce n'est pas comme un art autarcique, mais comme un espace public, inscrit dans l'histoire et dans le champ social. Aussi, si la sécheresse des registres peine à ressusciter la magie de la scène, "une série de dessins du temps nous permet de ressaisir quelque chose de la vision, sur les planches, de l'actrice, telle que la ville l'applaudissait ${ }^{83} »$. Il n'est de théâtre que dans l'espace social de la représentation et dans une interaction entre scène et salle, et l'information théâtrale n'a de prix qui si elle parvient à restituer ce que fut la vie théâtrale d'une époque ${ }^{84}$.

\footnotetext{
80 Esprit de Sophie Arnould, p. 9-10. Cette anecdote figure bien sûr dans le Paradoxe (p. 116), mais aussi dans l'Arnoldiana (p. 87), dans Sophie Arnould, comédie en trois actes par MM. Ad. De Leuven, De Forges et Ph. Dumanoir (Paris, Barba, 1833, I, 5, p. 9) et maints autres textes.

81 Sophie Arnould, p. 94 : Citant tous les rôles joués par l'actrice, les auteurs déclarent: «ce dépouillement des livrets des archives de l'Opéra ne présente que les rôles joués à Paris; il faudrait, pour avoir l'ensemble des services de la chanteuse donner ses créations sur les théâtres de la Cour, sur les théâtres de Versailles, de Choisy, de Fontainebleau ; il faudrait donner la liste fastidieuse des rôles indiqués par le Journal des spectacles de la Cour. Je ne veux pas le faire ».

82 Ibid., p. 117.

83 Ibid., p. 95

${ }^{84}$ Citant des vers de Rosalie Levasseur à l'encontre de Sophie Arnould, les Goncourt notent : " cette satire a un intérêt historique : elle donne le ton de l'engueulement de ce temps entre les étoiles de l'Académie royale de musique " (Sophie Arnould, p. 103). Cette ambition de faire non l'histoire du théâtre, mais celle de la vie théâtrale est sensible dans bien des ouvrages contemporains qui privilégient la dimension anecdotique de l'information.
} 
Au travers des vies d'actrices se dessine ainsi un petit théâtre du XVIII ${ }^{\mathrm{e}}$ siècle, qui laisse entrevoir dans la destinée féminine la vérité d'une époque, dont l'actrice est à la fois l'émanation et le symbole. Se faisant les historiens des maitresses de Louis XV, les Goncourt prévoyaient de faire à la femme « cette grande actrice méconnue de l'histoire, la place que lui a faite l'humanité moderne dans le gouvernement des mœurs et de l'opinion publique », afin de retrouver «ce grand secret d'un temps qui est la règle de ses institutions : l'esprit social $»^{85}$. Les actrices, comme les courtisanes, "méritent l'étude », dans la mesure où «elles font revivre leur patrie et leur temps. Elles avouent l'humanité tout entière d'Athènes, de Rome ou de Paris. Elles sont l'aimable et la franche confession des mours et des idées. Elles apportent avec leurs biographies la vie intime et déshabillée de la génération qu'elles enivrent ${ }^{86}{ }$. C'est en tant qu'emblèmes d'une époque, et non plus seulement d'un art, qu'elles offrent à l'historien une prise intéressante. Soucieux de « reconstruire un siècle [qu'ils aiment] ${ }^{87}$, les Goncourt ne sauraient rêver de sujet plus favorable que les actrices. Car le XIX ${ }^{\mathrm{e}}$ siècle voit dans le XVIII ${ }^{\mathrm{e}}$ le «siècle des comédiennes de haute lignée et des courtisanes royales» où «trois grandes comédiennes règnent plus que la femme de Louis XV et presque autant que ses maîtresses ${ }^{88}$ » et où « le rôle brillant qu'ont joué [...] les actrices, les chanteuses, les danseuses et les courtisanes de toutes classes, rappelle des événements singuliers qui ont influencé plus qu'on ne pense sur le système social ${ }^{89} »$. En ce sens Sophie Arnould, "courtisane la plus courtisane de l'époque la plus courtisane ", mérite bien une biographie ${ }^{90}$, de même que Clairon, ravalée à sa dimension de fille galante et devenue, à ce prix, l'incarnation d'un siècle délibérément revu et corrigé par les Goncourt.

Car, Barbey d'Aurevilly l'a bien noté, c'est en "courtisans » que les deux frères écrivent l'histoire de ces courtisanes, bien plus qu'en historiens ${ }^{91}$. Courtisans non pas des actrices, mais du siècle mythique dont elles seraient l'emblème. Le XVIII ${ }^{\mathrm{e}}$ siècle ressuscité sur le théâtre intime des vies d'actrice est en effet bien subjectif, et marqué par un certain nombre de refus: refus de l'idéalisation et du sentimentalisme, tout d'abord, qui transparait dans l'attention portée par les biographes à montrer l'envers du décor et les aspects scabreux de la « grande existence aux dessous de misère ${ }^{92}$ » des actrices (dettes, délabrement physique, maladies vénériennes... $\left.{ }^{93}\right)$; refus, également, du XVIII ${ }^{\mathrm{e}}$

${ }^{85}$ Les Maîtresses de Louis XV, OC, CL, p. 178.

86 Sophie Arnould, p. 14. Cette idée est commune au XIXe siècle. Voir Houssaye, Galerie de portraits du XVIII siècle, Paris, Charpentier, 1848, p. 435.

87 Portraits intimes du XVIII siècle, p. 7-12.

${ }^{88}$ Houssaye, La Comédie Française, p. 133.

89 Arnoldiana, p. 3-5.

90 Barbey d'Aurevilly, «Sophie Arnould », art. cit., p. 306.

${ }^{91}$ Ibid., p. 306.

${ }^{92}$ Mademoiselle Clairon, p. 100.

93 Ibid., p. 67-68 ; Sophie Arnould, p. 43 et 52. Barbey d'Aurevilly note que les Goncourt ne cherchent pas, à la différence d'autres historiens, à «blanchir le XVIII ${ }^{\mathrm{e}}$ siècle »: «Eux [...] 
siècle philosophique et sensible que ne cesse, dans ses Mémoires, de convoquer l'hypocrite Clairon. Ces refus, on l'a vu, ont pour fonction première d'évacuer des vies d'actrices tout ce qui relève du théâtre comme art, libérant le champ pour ce qui fait, aux yeux des Goncourt, le véritable théâtre de leur XVIII ${ }^{\mathrm{e}}$ siècle intime: l'esprit. C'est ce dernier qui explique leur prédilection pour Sophie Arnould et motive le projet de biographie la concernant ${ }^{94}$. Cet esprit n'est pas seulement celui de l'actrice, c'est «l'esprit de Paris, de la Comédie, d'une femme et d'une fille [...] soumettant les plus nobles oreilles au langage familier de la nature sans toilette ${ }^{95} »$. Dans une envolée lyrique, les Goncourt célèbrent ces «mots bondis de sa bouche» qui sont comme «l'écho et le testament libre du XVIII ${ }^{\mathrm{e}}$ siècle $!^{96} »$.

Aux côtés de Fontenelle, de Piron, de Voltaire, à qui elle est souvent associée dans les ana, Sophie Arnould incarne un siècle révolu, que le XIX ${ }^{\mathrm{e}}$ siècle ne peut plus évoquer que sur le mode de la nostalgie. Arsène Houssaye regrette «l'esprit du temps! le temps de l'esprit! - Ces mots d'outre-tombe [...] plus vivants que les mots de Ceritto. - Et de nos Chamfort et de nos Rivarol de 1850 : combien de nos beaux-esprits qui vivent des miettes de la table de Sophie Arnould $!^{97} »$. La même nostalgie est sensible chez Barbey d'Aurevilly: "Pendant des années, et jusqu'à la Révolution qui a tué l'esprit comme elle a tué tant d'autres choses, [Sophie Arnould] régna par les mots, la plus jolie manière de régner en France quand on y régnait $!^{98} "$ Avec la mort simultanée, en 1802, de Sophie Arnould, de Clairon et de Dumesnil, ce n'est donc pas seulement une génération d'actrices qui disparait, mais tout un monde ${ }^{99}$.

Dès lors, le projet des Actrices du XVIII siècle prend un autre sens et l'entreprise biographique des Goncourt, dont on a montré qu'elle n'était pas dénuée de partis pris, apparait, davantage que comme un essai historique, comme une tentative de résurrection. L'image est omniprésente sous la plume des Goncourt, qui devinent dans les documents autographe la «survie de l'homme », l'« immortalité des grandeurs et des misères de notre nature ${ }^{100}$. Ces actrices courtisanes, "qui, vivantes, sont le scandale d'un siècle, - et mortes, son sourire ${ }^{101}$ », ressuscitent sur le théâtre intime des Goncourt un

\footnotetext{
trouvent cette malpropreté piquante. Ils acceptent d'enthousiasme le dix-huitième siècle tout entier » (art. cit., p. 26).

94 Sophie Arnould, p. 11.

95 Ibid., p. 57.

96 Ibid., p. 59.

${ }_{97}$ Arsène Houssaye, Les Femmes du diable, p. ix.

98 Barbey d'Aurevilly, art. cit., p. 312.

99 Voir Houssaye, Les Femmes du diable, p. 290.

100 Portraits intimes du XVIII siècle, p. 7-12. Peut alors s'opérer une réconciliation entre Edmond et Clairon, unis par l'ambition commune de restituer la vérité d'une époque, dans le portrait historique pour l'un et le respect de la couleur locale dans le costume de scène pour l'autre (voir Mademoiselle Clairon, p. 82-83).

101 Sophie Arnould, p. 13.
} 
XVIII ${ }^{\mathrm{e}}$ siècle rêvé, irrémédiablement perdu. Le théâtre qui se déploie dans les pages des vies d'actrices, placé sous le signe de la féminité, de l'histoire intime et de l'esprit, n'est plus un théâtre de tréteaux et de toiles, mais un théâtre du fantasme. 
